\title{
A Competitive and Dynamic Pricing Model for Secondary Users in Infrastructure based Networks
}

\author{
Soumitra Dixit, Shalini Periyalwar, and Halim Yanikomeroglu \\ Broadband Communications and Wireless Systems (BCWS) Centre, \\ Department of Systems and Computer Engineering, Carleton University, Ottawa, Ontario K1S 5B6, Canada. \\ E-mail: \{sdixit, shalinip, halim\}@sce.carleton.ca
}

\begin{abstract}
Enabling unsubscribed Secondary User (SU) access through Dynamic Spectrum Access (DSA) techniques provides a huge opportunity to the Wireless Service Providers (WSPs) for achieving efficient radio spectrum usage as well as gain additional profits. This paper presents a novel and dynamic SU pricing model for implementation by the WSPs at their Base Stations (BSs) based upon a BS-centric distributed framework, that allows the SU price to vary dynamically with the changing radio spectrum usage at the BS. Assuming high competition among regional WSPs, a non-cooperative competition structure is considered among the WSPs, where neither information nor resources are shared among the WSPs. A simple single cell scenario easily scalable to multiple cells achieving competitive yet dynamic SU pricing among the BSs of two WSPs, is presented in this paper. The final $\mathrm{SU}$ price is seen to depend on the spectrum utilization at the BS, the wireless channel, and the price charged by WSPs to their Primary Users (PUs).
\end{abstract}

\section{INTRODUCTION}

Unbalanced utilization of the radio spectrum with respect to time and location coupled with huge investments by the Wireless Service Providers (WSPs) in acquiring the licensed spectrum, have a serious impact on the bottom line of the WSPs, and in turn on the price paid by the subscribers for wireless access. Enabling temporary wireless access to Secondary Users (SUs) through Dynamic Spectrum Access (DSA) techniques, provides the WSPs with an opportunity to not only improve the efficiency of radio spectrum usage but also earn additional profits from such SU access [1], [2].

To this effect, majority of the work in literature has focused on spectrum sharing techniques and SU pricing models based on a centralized approach, where a Centralized Mediating Entity (CME) such as a spectrum broker or a spectrum regulator pools the unutilized spectrum information from all the WSPs in the area [1], [3]-[5]. This approach is envisioned to regulate, co-ordinate and lease the spectrum temporarily (hours) to either WSPs interested in temporarily increasing their capacity [3], [4], or directly to SUs requiring temporary wireless access [5], [6].

However, two primary difficulties can be foreseen considering the implementation of the centralized approaches described above involving CMEs. Firstly, radio spectrum usage characteristics are observed to vary with respect to time and location, thus leading to the requirement of multiple regional CMEs with increased signaling overhead for CME-WSP coordination. Secondly, considering the highly competitive multi-
WSP environment, it can be considered unlikely for the WSPs to share their spectrum or spectrum related information.

In this paper, we present a methodology to implement the Base Station (BS)-centric distributed framework from [2] for achieving competitive yet dynamic SU pricing among the WSPs in a region, while simultaneously maximizing individual WSP profits from SU access. Since the analysis in this paper is based on the BS-centric distributed framework from [2], the individual WSPs are assumed to operate without the presence of CMEs, and in a non-cooperative manner without any sharing of spectrum or spectrum related information. It must be noted that, the BSs are considered to be independent elements setting SU prices based on their local spectrum utilization, and act in the best interest of their respective WSPs.

The organization of this paper is as follows: Section II introduces the BS-centric distributed framework and the dynamic incentive based SU pricing model [2]. Section III describes the methodology for achieving profitable yet competitive dynamic SU pricing among the WSPs in the area, without inter-WSP cooperation. Simulations and results showcasing the profitability potential to the WSPs from such SU access are presented in Section IV, with Section V highlighting the conclusions.

\section{BS-CEnTRiC Distributed FrameWORK AND DYNAMIC SU PRICING}

The BS-centric distributed framework [2] implemented in this paper considers enhanced BSs with the capability of providing direct temporary SU access, by conducting SU admission control and Primary User (PU)-SU resource allocation decisions based on their instantaneous spectrum usage characteristics. This distributed framework not only improves the instantaneous spectrum usage, but also reduces the heavy signaling overhead required in CME-WSP-BS coordination.

Every BS in this distributed framework broadcasts their local spectrum information (e.g., SU price per application class) to the SUs in the area. The SUs can therefore select the mostsuitable BS and potentially acquire temporary wireless access instantaneously directly from the BS, rather than waiting for the CME to negotiate and select a WSP, which in turn selects the most suitable BS to provide access to the SU. It must be noted that, the BS-centric distributed framework [2] allows temporary wireless access to SUs, if and only if, there remain surplus spectrum available at the BS after the all PUs (i.e., 


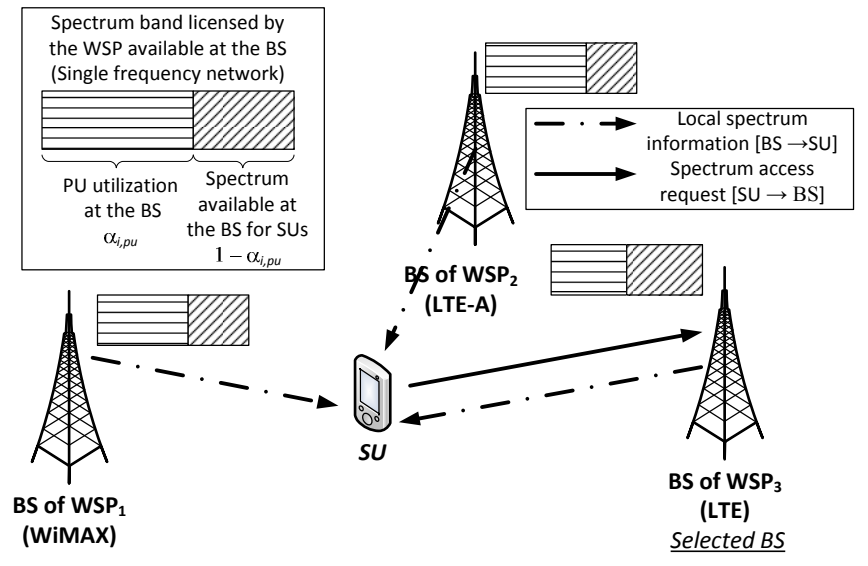

Fig. 1. Distributed framework for SU access [2]

subscribers) have been satisfactorily served, thus guaranteeing no disruption to PU service.

Figure 1 illustrates the BS-centric distributed framework based scenario, where a SU can potentially gain temporary wireless access from the BS of one of the three WSPs. The WSPs are assumed to have a single frequency network with an all-IP framework, where the unutilized spectrum at each $\mathrm{BS}$ can be made available to the SU in its coverage area.

\section{A. Terminology: SU Pricing Model}

Assuming the total spectrum at the BS to be 1 unit, the fraction of the spectrum utilized by PUs at $\mathrm{BS}_{i}$ is denoted by $\alpha_{i, p u}$, while the unutilized spectrum available to the SUs is given by $\left(1-\alpha_{i, p u}\right)$, as shown in Fig. 1. The variable price charged to the SUs by the BSs (WSPs) for a particular application class (finite time $t$ in seconds: delay sensitive applications, finite block of $B$ bytes: delay insensitive applications) is known as the SU price and denoted by $s_{i}$. The fixed price charged by the WSPs to the PUs for the same application class is referred to as the PU price denoted by $p_{i}$.

Before detailing the SU pricing model, the terminology relevant to spectrum utilization at $\mathrm{BS}_{i}$ is listed as follows:

$\alpha_{i, p u}:$ PU utilization or PU demand at $\mathrm{BS}_{i}$,

$\alpha_{i, h}$ : Spectrum reserved (handoff and overload protection),

$\alpha_{i, t h}:$ Threshold for spectrum utilization; $\alpha_{i, t h}=1-\alpha_{i, h}$,

$\alpha_{i, s u}$ : SU utilization; $\alpha_{i, s u}$ exists iff $\alpha_{i, p u}<\alpha_{i, t h}$,

$\alpha_{i, t}$ : Spectrum Utilization Factor (SUF), i.e., $\alpha_{i, p u}+\alpha_{i, s u}$,

$\alpha_{i, i c}$ : Incentive cutoff limit beyond which $s_{i}>p_{i}$,

where $\alpha_{i, p u}, \alpha_{i, h}, \alpha_{i, t h}, \alpha_{i, s u}, \alpha_{i, t}, \alpha_{i, i c} \in[0,1]$.

\section{B. Dynamic Incentive based Pricing Model}

The SU price $s_{i}$ is defined in terms of the fixed PU price $p_{i}$ and the SUF at the BS can be given as [2]

$$
s_{i}=\left(f_{i}\left(\alpha_{i, t}\right)\right)^{m_{i}} \times p_{i},
$$

where $s_{i}, f_{i}\left(\alpha_{i, t}\right), p_{i}$ and $m_{i}$ are non-negative real numbers.
The term $f_{i}\left(\alpha_{i, t}\right)$ is the normalized SU price with respect to (w.r.t.) the fixed $p_{i}$ and captures the variable nature of spectrum utilization at the BS. This term based on the log barrier function [7], and has an inherent property for SU admission admission control. The normalized SU price is defined as

$$
f_{i}\left(\alpha_{i, t}\right)= \begin{cases}-\ln \left(1-\left(\frac{\alpha_{i, t}}{\alpha_{i, t h}}\right)^{n_{i}}\right) & \text { if } \alpha_{i, t}<\alpha_{i, t h} \\ \infty & \text { if } \alpha_{i, t} \geq \alpha_{i, t h}\end{cases}
$$

where $n_{i}$ is a positive real number representing the Incentive Cutoff Factor (ICF). The ICF is useful in defining the incentive in (2), such that when $\alpha_{i, t}<\alpha_{i, i c}$, we have $s_{i}<p_{i}$. The value of ICF is be obtained by solving (2) setting $\alpha_{i, t}=\alpha_{i, i c}$ and $f_{i}\left(\alpha_{i, t}\right)=1$ [2]. This incentive structure embedded into the SU pricing model, depends on the PU price, and allows the WSPs to attract more SUs during periods of low PU demand (off-peak hours) and discourages the SUs to join the network during periods of high PU demand (peak hours).

The exponent of $f_{i}\left(\alpha_{i, t}\right)$ in (1) is a non-negative real number referred to as the Price Leveling Factor (PLF) denoted by $m_{i}$, and provides additional flexibility to the WSPs for pricing the SUs. The PLF is used in the next section to set SU prices with the aim of maximizing individual WSP profits, considering inter-WSP competition and SU pricing incentives.

Assuming $\alpha_{i, p u}<\alpha_{i, i c}$, Fig. 2 shows the dynamic nature of the pricing model for $\mathrm{BS}_{i}$ with the configuration parameters $\alpha_{i, i c}=0.7$ and $\alpha_{i, t h}=0.9$. The original SU price with PLF $m_{i}=1$ is denoted by $s_{i}$, while the new adjusted SU pricing curve with PLF $m_{i} \neq 1$ is denoted as $s_{i}^{\prime}$ in Fig. 2 . The profits from SUs can be demonstrated comparing the PLF $m_{i}=1$ and $m_{i}=0.3$, with the latter assuring profits to the WSP for every SU served considering a fixed cost $C$.

The pricing model [2] above can be described as dynamic and opportunistic. Dynamic nature of the pricing model can be attributed to the SU price changing w.r.t. the SUF at the

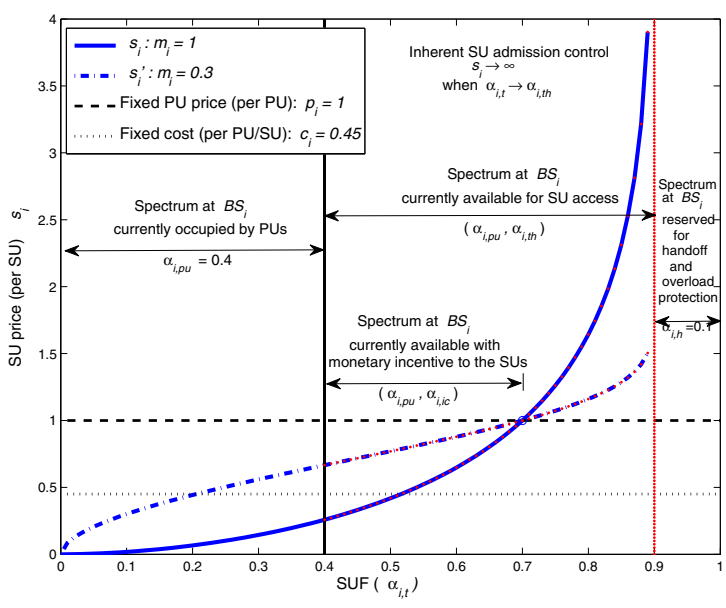

Fig. 2. Incentive based dynamic model for SU pricing [2] WSP configuration parameters $\alpha_{i, i c}=0.7, \alpha_{i, t h}=0.9$ 
$\mathrm{BS}_{i}$. The SU pricing structure is calculated at $\mathrm{BS}_{i}$ using (1) for a fixed time window $T$ (e.g., 10 minutes) based on the predicted PU demand, and is then broadcasted to the SUs in the area. The SUs gaining access during this time window are priced opportunistically with the SU price being the lowest for the first SU and incrementing for every subsequent SU.

\section{Multiple WSPs and Competitive Pricing}

Considering PU service, the PUs pay a fixed price (per month, minute, bytes) for a given service (e.g., voice or data) to the WSPs. With multiple WSPs competing (no cooperation among WSPs) for a larger subscriber base, the PU prices set by each WSP can be seen to stabilize and fall into equilibrium, thus maximize individual WSP profits and providing no incentives to the WSPs for deviating from the set prices [8].

Efficient spectrum utilization requires the WSPs not only to maximize their subscriber base (PUs), but also attract SUs to gain temporary access to the unutilized spectrum at their respective BSs. The WSPs would thus would compete with each other in a non-cooperative manner, with the goal of setting SU prices to maximize their individual profits from SU access. The dynamic SU pricing model allows the WSPs to provide incentives in price to attract the SUs during periods of low SUF, i.e., $\alpha_{i, p u}<\alpha_{i, t}<\alpha_{i, i c}$.

Although the PLF $\left(m_{i}\right)$ provides flexibility to the WSPs to adjust their $\mathrm{SU}$ prices, obtaining equilibrium pricing among the WSPs with dynamically changing prices could be prohibitively complex. Therefore a methodology for achieving inter-WSP competitive yet dynamic SU pricing is developed in the next section, based on the linear city model and static SU pricing.

\section{Non-Cooperative Competitive SU Pricing}

Assuming the BSs as individual entities acting on behalf of their respective WSPs, we consider a single cell scenario to initially find equilibrium SU prices considering static SU pricing. Calculation of these static equilibrium SU prices based on the linear city model will be the intermediate step in achieving non-cooperative competitive dynamic SU pricing.

\section{A. Linear City Model}

The linear city model assumes a city to be represented by a line of distance 1 unit with a single BS for each of the two competing WSPs placed on either side of the city, as shown in Fig. 3. Since each of the two WSPs in the linear city scenario have only one BS, the same index $i$ will be used for the WSPs, beyond this point in the paper. The unit distance on the linear city is represented by $y \in[0,1]$, and the BSs are assumed to be at a distance $d f_{i}$ from the city, where $d f_{i}$ is the exclusion region and depends on antenna configurations at the BS of $\mathrm{WSP}_{i}$. Hence the linear city extends from $d f_{1}(y=0)$ to the cell edge $(y=1)$ for $\mathrm{WSP}_{1}$ and vice versa for $\mathrm{WSP}_{2}$.

For static SU pricing structure, we assume a fixed SU price $S_{i}$ to be charged by the WSPs to all the SUs, such that the SUs have a monetary incentive w.r.t. the PU price, i.e., $S_{i}<p_{i}$. The cost to each WSP for providing temporary wireless access to a single $\mathrm{SU}$ is assumed to be fixed and equal $C=C_{1}=$

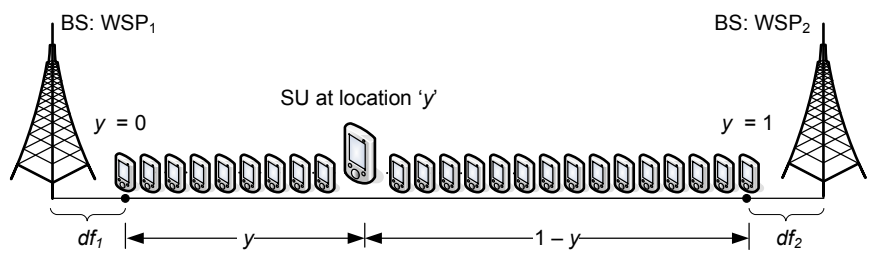

Fig. 3. SU service differentiation based on the linear city model

$C_{2}$. Thus from the profitability perspective of the WSPs, the equilibrium SU price $S_{i}^{*}$ must satisfy $C_{i}<S_{i}^{*}<p_{i}$. It must be noted that the SU prices $\left(S_{1}, S_{2}\right)$ are assumed to be set simultaneously by the WSPs with the goal of individual profit maximization, without inter-WSP cooperation.

Price based competition among multiple sellers (i.e. WSPs) theoretically leads to equilibrium prices equaling to the costs, i.e., $S_{i}^{*}=C_{i}$, thus resulting in zero profits to the sellers [8], [9]. However, such a phenomenon is rarely observed in real markets, since the sellers always distinguish their service based on quality, brand, promotional offers, etc.. The differentiation of their own service from similar service by other WSPs in the region, creates a niche market for the WSPs, maximizing their individual profits as well as competing on prices.

The methodology for achieving competitive inter-WSP SU pricing is thus based on this intermediate step of fixed equilibrium pricing analysis with $\mathrm{SU}$ service differentiation based on the linear city model. The PLF $\left(m_{i}\right)$ introduced in Section II can then be used to transform the fixed equilibrium SU prices into competitive yet dynamic SU prices.

A total of $q$ SUs are assumed to be uniformly distributed along the linear city from 0 to 1 , and each $\mathrm{SU}$ is required to select either of the two WSPs for temporary access. The BSs of the two WSPs are assumed to have sufficient unutilized spectrum in total for serving all the SUs on the linear city.

From the SU's perspective, selection of a WSP not only depends on the price but also on the signal strength at the SU terminal. Therefore the perceived price (monetary unit: '\$') for a SU located at any point ' $y$ ' can be given as [9]

$$
U_{i}(y)=S_{i}+(\zeta \times y)
$$

where $\zeta$ is a constant nonnegative real number representing the dissatisfaction level of a SU. Observing (3), the perceived price to the $\mathrm{SU}$ for access to the $\mathrm{BS}$ of $\mathrm{WSP}_{i}$ and hence the dissatisfaction level can be seen to increase as $y$ increases.

\section{B. SU Dissatisfaction Level $\zeta$}

In order to define the SU dissatisfaction level $\zeta$ in (3), we first define the Satisfaction Level (SL) denoted by $\tau_{i}$ for the SUs w.r.t. the $\mathrm{BS}$ of $\mathrm{WSP}_{i}$. The SL $\tau_{i}(r)$ for the $r^{t h} \mathrm{SU}$ on the linear city w.r.t. the $\mathrm{BS}$ of $\mathrm{WSP}_{i}$ is defined in terms of the spectral efficiency $\eta_{i}(r)$ at the SU terminal as

$$
\tau_{i}(r)=\frac{\eta_{i}(r)}{\max \left(\eta_{i}\right)} .
$$

Averaging out the shadowing, the maximum value of the spectral efficiency $\eta_{i}$ can be observed at $d f_{i}$, i.e., at $y=0$ 
nearest to BS of $\mathrm{WSP}_{i}$. The standard deviation of the SLs from (4) is denoted by $\sigma_{i}$, and can be used to represent the wireless channel on the linear city. The dissatisfaction level $\zeta$ can be considered as the reciprocal of the SL and can be defined as:

$$
\begin{gathered}
\zeta=K_{1} K_{2} \quad(\$), \\
\text { where } K_{1}=\frac{1}{\Delta\left(K_{2}\right)}(\$) \text { and } K_{2}=\left(\frac{\sigma_{1}+\sigma_{2}}{2}\right) .
\end{gathered}
$$

$K_{1}$ and $K_{2}$ are constants and nonnegative real numbers, and $\Delta\left(K_{2}\right)$ defines the unit change in the value of $K_{2}$. With $\zeta$ defined, we now proceed to calculate the equilibrium $\mathrm{SU}$ prices with a static SU price $\left(S_{i}\right)$ and fixed equal cost $C$.

\section{Equilibrium Analysis: Static SU Pricing}

For any SU located at a distance $y$ from $\mathrm{BS}$ of $\mathrm{WSP}_{1}$ as shown in Fig. 3, the SU will select $\mathrm{WSP}_{1}$ if $U_{W S P, 1}(y)<$ $U_{W S P, 2}(1-y)$, while it will select $\mathrm{WSP}_{2}$ if $U_{W S P, 1}(y)>$ $U_{W S P, 2}(1-y)$, and in case $U_{W S P, 1}(y)=U_{W S P, 2}(1-y)$ either of the two WSPs is randomly selected [9].

It could be possible for $\mathrm{WSP}_{1}$ to capture the entire market by setting $S_{1}$ such that $S_{1}<S_{2}-\zeta$, i.e., serve all the $q$ SUs in the linear city. However, in context of the system framework and the assumptions described, due to limited quantity of spectrum in offer for the SUs, the entire SU demand cannot be satisfied by a single WSP. Also $\mathrm{WSP}_{1}$ would need to know the SU price $S_{2}$ set by $\mathrm{WSP}_{2}$, which is contrary to our assumptions that $\mathrm{SU}$ prices are set simultaneously.

Therefore, to achieve competitive SU pricing, the WSPs will need to set their SU prices close to each other. From the SUs' perspective, the SUs will prefer to obtain access from the WSP with the lower SU price, since the SUs cannot be guaranteed Quality of Service (QoS) at all times as the BSs are required to allocate resources to satisfactorily serve the PUs first [2]. With the SU prices set to be close (or equal) to each other by the WSPs, the SUs will prefer to select the WSP with the lower perceived price thus gaining a higher SL.

We now focus on a SU located at a distance $d$ on the linear city, such that $U_{W S P, 1}(d)=U_{W S P, 2}(1-d)$. Solving the equality for $d$, gives us the $\mathrm{SU}$ demand at $\mathrm{WSP}_{1}$ as

$$
D_{1}\left(S_{1}, S_{2}\right)=\frac{\zeta-S_{1}+S_{2}}{2 \zeta},
$$

where $D_{1}\left(S_{1}, S_{2}\right)$ is a nonnegative real number. The profit achievable by $\mathrm{WSP}_{1}$ based on SU demand can be given as

$$
\pi_{1}\left(S_{1}, S_{2}\right)=S_{1} \times\left(D_{1}\left(S_{1}, S_{2}\right)\right)-C \times\left(D_{1}\left(S_{1}, S_{2}\right)\right),
$$

where $\pi_{1}\left(S_{1}, S_{2}\right)$ is a nonnegative real number. For profit maximization, $\mathrm{WSP}_{1}$ 's best response to the $\mathrm{SU}$ price set by $\mathrm{WSP}_{2}$ can be obtained as

$$
B R_{1}\left(S_{2}\right)=\frac{\partial \pi_{1}}{\partial S_{1}}=\frac{S_{2}+\zeta+C}{2} .
$$

The model being symmetric for both the WSPs, a similar set of results can be obtained for $\mathrm{WSP}_{2}$. Thus assuming $S_{1}=S_{2}$, the Nash Equilibrium (NE) SU price can be expressed as

$$
S^{*}=C+\zeta,
$$

where $S^{*}$ is non-negative real number [9].

\section{Transformation to Dynamic SU Pricing}

The PLF [2] described in Section II provides additional price adjustment flexibility to the incentive based dynamic pricing model, and therefore can be used to transform the static NE SU price into dynamic yet competitive SU pricing.

To implement the transformation, initially, the SU price to be paid by the first $\mathrm{SU}$ is adjusted to be equal to the static NE SU price obtained from (9). The value of the PLF is obtained next by rearranging (1) and setting $s_{i}=S_{i}^{*}$ and $\alpha_{i, t}=\alpha_{i, p u}$. Finally, the PLF is substituted in (1) to obtain the new dynamic yet competitive SU pricing structure denoted by $s_{i}^{\prime}$.

Thus it can be observed that incremental SU prices are charged to every new SU, depending upon the amount of unutilized spectrum available at the BS. Since the NE SU price is adjusted to be equal to SU price of the first $\mathrm{SU}$ and every subsequent SU pays a higher SU price, the WSPs are guaranteed profits from every SU.

\section{Simulations And Results}

For analyzing the profitability potential for the WSPs, a single cell with no inter-cell interference is assumed for the linear city. The wireless channel is based on the urban macrocell scenario [10], and the simulation parameters are defined in Table I. The carrier frequency of $2 \mathrm{GHz}$ for $\mathrm{WSP}_{1}$ and $1.9 \mathrm{GHz}$ for $\mathrm{WSP}_{2}$ is assumed giving a path loss of $P L_{1}=128.1+$ $37.6 \log _{10}(\delta)$ and $P L_{2}=127.7+37.6 \log _{10}(\delta)$ respectively, where $\delta$ is the distance in meters. Every SUs entering $\mathrm{BS}_{i}$ is randomly allocated either 400,500 or $600 \mathrm{KHz}$, assuming the total bandwidth at $\mathrm{BS}_{i}$ to be $20 \mathrm{MHz}$.

The simulation scenario considered in this paper assumes similar PU utilization at the BSs of the two WSPs and with symmetric incremental WSP costs. However, it must noted that, other scenarios with dissimilar PU utilization and asymmetric WSP costs can also be shown to achieve high inter-WSP competitive pricing.

The competitive nature of the dynamic SU prices can be quantified using the following inter-WSP competitiveness metric:

$$
\psi_{s_{1}, s_{2}}=\operatorname{VAR}(\Lambda) \text {, where } \Lambda=\left|\hat{s}_{1}-\hat{s}_{2}\right|,
$$

and VAR denotes the variance. $\hat{s}_{1}=\left\{s_{1}(1), s_{1}(2), \ldots, s_{1}\left(L_{1}\right)\right\}$ and $\hat{s}_{2}=\left\{s_{2}(1), s_{2}(2), \ldots, s_{2}\left(L_{2}\right)\right\}$ give the vector of the

TABLE I

SIMULATION PARAMETERS

\begin{tabular}{|c|c|}
\hline \hline Parameter & WSP $_{1}$ or $_{\text {WSP }}$ \\
\hline BS-cell edge distance & $250 \mathrm{~m}$ \\
\hline Exclusion distance $d f_{i}$ & $35 \mathrm{~m}$ \\
\hline BS total transmit power & $46 \mathrm{dBm}$ \\
\hline BS transmitter antenna gain & $14 \mathrm{~dB}$ \\
\hline SU terminal receiver antenna gain & $0 \mathrm{~dB}$ \\
\hline Noise figure & $5 \mathrm{~dB}$ \\
\hline Noise power density at SU terminal & $-174 \mathrm{dBm} / \mathrm{Hz}$ \\
\hline Target BER & $10^{-6}$ \\
\hline
\end{tabular}




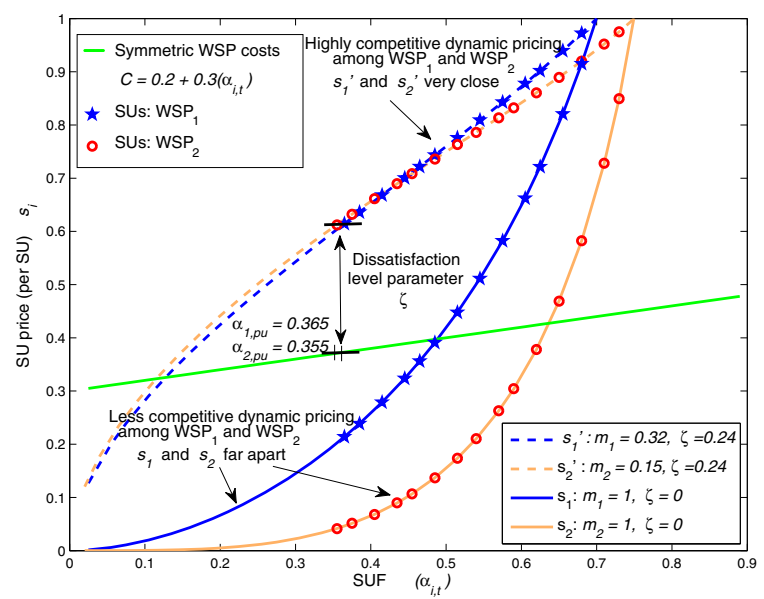

Fig. 4. Competitive nature of SU pricing among $\mathrm{WSP}_{1}$ and $\mathrm{WSP}_{2}$ WSP configuration parameters specified in Table II

original $\mathrm{SU}$ prices set by $\mathrm{WSP}_{1}$ and $\mathrm{WSP}_{2}$ respectively, while the vector of the new SU prices is given by $\hat{s}_{i}^{\prime}$. Positive integers $L_{1}$ and $L_{2}$ represent the number of SUs served by the $\mathrm{WSP}_{1}$ and $\mathrm{WSP}_{2}$ respectively. Since either $L_{1} \geq L_{2}$ or $L_{1} \leq L_{2}$ such that $q=L_{1}+L_{2}$, we concatenate either $\hat{s}_{1}$ or $\hat{s}_{2}$ such that $L_{1}=L_{2}$ before using them in (10).

The competitive nature with dynamic SU pricing can be observed in Fig. 4, with the new SU price $\left(s_{i}^{\prime}\right)$ curves for both WSPs seen to be very close to each other, in comparison to the original SU price $\left(s_{i}\right)$ curves. The lower value of the metric $\psi_{s_{1}^{\prime}, s_{2}^{\prime}}$ for the new SU prices w.r.t. $\psi_{s_{1}, s_{2}}$ for the original SU prices as in Table II, validates the competitive nature of the new SU prices. High inter-WSP competition and Cumulative Profits (CP) for the new SU prices can be seen in Fig. 5.

It must be noted that the $\mathrm{SU}$ profit results shown in table II and Fig. 5 consider only one BS for the time window $T$,

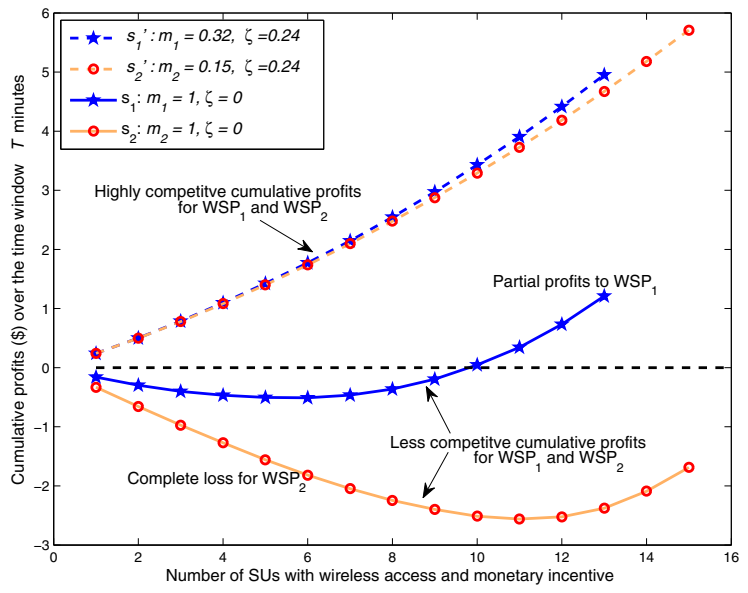

Fig. 5. Cumulative profits to WSPs from SUs WSP configuration parameters specified in Table II
TABLE II

WSP PARAMETERS AND RESULTS

\begin{tabular}{|c|c|c|}
\hline \hline Parameter & $\mathbf{W S P}_{1}$ & $\mathbf{W S P}_{2}$ \\
\hline PU utilization $\alpha_{i, p u}$ & 0.365 & 0.355 \\
\hline Spectrum threshold $\alpha_{i, t h}$ & 0.9 & 0.85 \\
\hline Incentive cutoff limit $\alpha_{i, i c}$ & 0.7 & 0.75 \\
\hline Incentive cutoff factor $n_{i}$ & 1.83 & 3.67 \\
\hline Results & $\mathbf{W S P}_{1}$ & $\mathbf{W S P}_{2}$ \\
\hline Number of SUs served with monetary incentive & 13 & 15 \\
\hline $\mathrm{CP}$ with $\zeta=0.24(\$)$ & 4.95 & 5.71 \\
\hline$\%$ rise in CP for $s_{i}^{\prime}$ w.r.t. $s_{i}$ & 79.53 & 129.54 \\
\hline \hline Competitiveness $\mid \psi_{s_{1}^{\prime}, s_{2}^{\prime}}: 3.44 \times 10^{-4}$ & $\psi_{s_{1}, s_{2}}: 5.24 \times 10^{-2}$ \\
\hline \hline
\end{tabular}

and SUs joining the network only when incentives in prices are available. However, this model can be easily scaled to estimate the overall daily SU profit to the WSPs, by simply multiplying the $\mathrm{SU}$ profits obtained from a single $\mathrm{BS}$ in a day with the total number of BSs owned by the WSP.

\section{CONCLUSIONS}

This paper provides a methodology for achieving noncooperative competitive yet dynamic SU pricing, based on a novel BS-centric distributed framework. The framework and methodology described in this paper demonstrates the profitability potential to the WSPs from SU access based on the distributed framework. The final competitive dynamic SU price set by the BS for direct temporary wireless access to the SUs can be observed to depend upon the wireless environment, the SUF at the BS, current PU demand and the PU price.

\section{REFERENCES}

[1] T. Weiss and F. Jondral, "Spectrum pooling: an innovative strategy for the enhancement of spectrum efficiency," IEEE Communications Magazine, vol. 42, no. 3, pp. S8-14, March 2004.

[2] S. Dixit, S. Periyalwar, and H. Yanikomeroglu, "A distributed framework with a novel pricing model for enabling dynamic spectrum access for secondary users," in Proc. IEEE VTC 2009-Fall.

[3] M. Buddhikot, P. Kolodzy, S. Miller, K. Ryan, and J. Evans, "Dimsumnet: new directions in wireless networking using coordinated dynamic spectrum," in Proc. of WoWMoM, June 2005, pp. 78-85.

[4] D. Niyato and E. Hossain, "Microeconomic Models for Dynamic Spectrum Management in Cognitive Radio Networks ", in Cognitive Wireless Communication Networks, E. Hossain and V.K. Bhargava, Ed. US: Springer, 2007, pp. 391-423.

[5] O. Ileri, D. Samardzija, and N. Mandayam, "Demand responsive pricing and competitive spectrum allocation via a spectrum server," in Proc. IEEE DySPAN 2005, pp. 194-202.

[6] J. Perez-Romero, O. Salient, R. Agusti, and L. Giupponi, "A novel ondemand cognitive pilot channel enabling dynamic spectrum allocation," in Proc. of IEEE DySPAN, April 2007, pp. 46-54.

[7] S. Boyd and L. Vandenberghe, Convex Optimization. Cambridge, U.K: Cambridge University Press, 2004.

[8] R. Barnes, Economic Analysis, An Introduction. London, U.K: Butterworth \& Co. Ltd., 1971.

[9] B. Polak, Game Theory Lecture Notes. Yale University open courses, 2007. [Online]. Available: http://oyc.yale.edu/economics/game-theory/

[10] 3GPP TR 36.942 v1.2.0, "E-UTRA Radio Frequency (RF) system scenarios (Release 8)," June 2007. 